\title{
Uso de dentífricos y colutorios para el control de la hipersensibilidad dentinaria en adultos: una revisión narrativa.
}

\section{Use of toothpastes and mouthwashes for the control of dentin hypersensitivity in adults: a narrative review.}

\author{
Catalina Moreno-Rabie ${ }^{1^{*}}$, Daniel Scharager-Lewin ${ }^{2}$, Vicente Aranguiz ${ }^{3}$
}

1. Universidad de los Andes, Santiago, Chile. Cirujano dentista

2. Universidad de los Andes, Santiago, Chile.

Periodoncia

3. Universidad de Los Andes, Santiago, Chile.

* Correspondencia autor: Catalina Moreno-Rabie | Parque los castaños 1710 casa 29, Santiago, Chile | E-mail: cmoreno@miuandes.cl

Trabajo recibido el 10/08/2017.

Aprobado para su publicación el 07/10/2017

\section{RESUMEN}

Objetivo: Describir la efectividad del uso de los distintos agentes desensibilizantes contenidos en dentífricos y colutorios para el control de la hipersensibilidad dentinaria en adultos.

Método: Se realizó una búsqueda electrónica de la evidencia científica en las bases de datos PubMed, Epistemónikos, EBSCO, BEIC y Cochrane Library; utilizando una estrategia de búsqueda que incluye las palabras clave. Para cada artículo encontrado se analizó el nivel y calidad de evidencia, riesgo de sesgo y ética. Resultados: En total 36 artículos fueron incluidos, 29 ensayos clínicos controlados aleatorizados y 7 revisiones sistemáticas. De los ensayos clínicos encontrados, cinco probaron la eficacia de colutorios, dos realizaron una comparación con dentífricos y colutorios y 22 evaluaron sólo el uso de dentífricos. Los agentes desensibilizantes testeados principalmente encontrados fueron sales de potasio, arginina, nano-hidroxiapatita, fosfosilicato de calcio y sodio (CSFS) y sales de estroncio. Sólo los primeros dos agentes fueron evaluados en presentación dentífrico y colutorio. Todos los ensayos clínicos demostraron reducir la hipersensibilidad dentinaria con alguno de los agentes utilizados en comparación a la medición inicial. Conclusión: Se podría recomendar el uso de nitrato y citrato de potasio, arginina, fosfosilicato de calcio y sodio y nano-hidroxiapatita en dentífricos para el tratamiento de la hipersensibilidad.

PALABRAS CLAVE:

Hipersensibilidad dentinaria; Sensibilidad dentinaria; Sensibilidad dental.

Rev. Clin. Periodoncia Implantol. Rehabil. Oral Vol. 11(3); 177-183, 2018.

\section{ABSTRACT}

Objectives: Describe the effectiveness of the use of different desensitizing agents contained in dentifrices and mouthwashes for the control of dentin hypersensitivity in adults. Method: An electronic search of the scientific evidence available was made in PubMed, Epistemónikos, EBSCO, BEIC and Cochrane Library; using a search strategy that included the key words. For each article found, the level and quality of evidence, risk of bias and ethics were analyzed. Results: A total of 36 articles were included, 29 randomized controlled clinical trials and 7 systematic reviews. Of the clinical trials, five tested the efficacy of mouthwashes, two made a comparison using dentifrices and mouthwashes and 22 tested only the use of dentifrices. The desensitizing agents mainly found were potassium salts, arginine, nano-hydroxiapatite, calcium sodium phosphosilicate (CSPS) and strontium salts. Only the first two were tested in both presentations. All the clinical trials demonstrated a reduction in dentin hypersensitivity with the use of an agent in comparison to the initial measurement. Conclusions: The use of nitrate and citrate potassium, arginine, calcium sodium phosphosilicate and nanohydroxyapatite in dentifrices for the treatment of hypersensitivity may be recommended. KEY WORDS:

Dentin hypersensitivity; Dentin sensitivity; Tooth sensitivity.

Rev. Clin. Periodoncia Implantol. Rehabil. Oral Vol. 11(3); 177-183, 2018.

\section{RELEVANCIA CLÍNICA}

La hipersensibilidad dentinaria es un dolor dentario de alta prevalencia en la población. Su manejo debiese corresponder a un conocimiento básico para el quehacer odontológico. Para mejorar la calidad de vida de los pacientes y tratando de mantener un enfoque preventivo y de terapia no invasiva se recomienda el uso de dentífricos y colutorios. Existiendo una gran variedad de productos disponibles en el mercado se plantea la interrogante acerca de cuál de ellos es realmente efectivo a corto y largo plazo y cuál de los agentes es mejor que otro. 


\section{INTRODUCCIÓN}

La hipersensibilidad dentinaria (HSD) se define como un dolor corto y agudo que surge de la dentina expuesta en respuesta a estímulos, generalmente térmicos, evaporativos, táctiles, osmóticos o químicos, que no puede ser asociado a cualquier otro defecto dental o enfermedad(1). Tiene una prevalencia variable. Según el consenso Canadiense sobre el manejo de la hipersensibilidad dentinaria en el año 2003, se habla de una prevalencia entre $3-57 \%$ en la población general ${ }^{(1)}$ y $23-80 \%$ post terapia periodontal| ${ }^{(2)}$.

El diagnóstico de la hipersensibilidad es complejo, pues la pulpa no responde de igual manera a los estímulos mecánicos o táctiles, químicos, eléctricos, evaporativos y térmicos ${ }^{(3,4)}$. Por esta razón, se recomienda utilizar al menos dos estímulos hidrodinámicos para su identificación acompañados de una escala subjetiva del dolor ${ }^{(5)}$, además que es esencial el diagnóstico diferencial porque por definición la HSD es un diagnóstico por exclusión ${ }^{(1)}$.

Las distintas estrategias para su tratamiento convergen en que la terapia debe orientarse desde la educación y prevención de factores de riesgo, hasta la realización de acciones invasivas como restauraciones o tratamiento quirúrgico ${ }^{(1,6)}$. Entre estas estrategias, encontramos los tratamientos de uso en casa como los dentífricos y colutorios.

Los agentes desensibilizantes, contenidos en dentífricos y colutorios, actúan mediante dos mecanismos: desensibilizando el terminal nervioso e impidiendo la transmisión del impulso u ocluyendo los túbulos dentinarios expuestos $^{(6-8)}$. Existen abundantes agentes destinados a aliviar la sintomatología, sin embargo, no se ha determinado el gold standard para el tratamiento ${ }^{(9)}$

Frente a esta problemática se planteó la siguiente pregunta de investigación: ¿Cuál es la efectividad clínica de los distintos agentes desensibilizantes contenidos en dentífricos y colutorios en el control de la hipersensibilidad dentinaria en adultos?

Siendo el objetivo principal de este estudio describir la efectividad del uso de los agentes desensibilizantes contenidos en dentífricos y colutorios para el control de la hipersensibilidad dentinaria en adultos.

\section{MATERIAL Y MÉTODO}

\section{Criterios de inclusión}

- Publicaciones como ensayos clínicos controlados aleatorizados, revisiones sistemáticas y meta-análisis.

- Publicaciones sin límite de idiomas.

- Artículos disponibles en texto completo.

- Estudios en que la población evaluada sea mayor de 18 años.

- Estudios cuya intervención sea un dentífrico o colutorio.

- Estudios que utilicen al menos dos métodos diagnósticos para evaluar la HSD.

\section{Criterios de exclusión:}

- Estudios en que el control o comparador sea distinto a un placebo, dentífrico o colutorio.

- Estudios que no describan el diagnóstico clínico de la HSD.

Para la selección y fundamento de los criterios de inclusión y exclusión, se utilizó como guía un consenso de expertos para el desarrollo de ensayos clínicos de hipersensibilidad dentinaria publicado por Holland, et al. en $1997^{(5)}$.

Se realizó una búsqueda de la evidencia disponible hasta el 18 de junio de 2016, en las siguientes bases de datos: PubMed, PubMed Clinical Queries, Epistemonikos, EBSCO, BEIC y Cochrane Library.

Para la recopilación de los artículos se realizó una estrategia de búsqueda electrónica que contenía las siguientes palabras clave: "dentin hypersensitivity", "dentin sensitivity", "tooth sensitivity", combinado con los términos "therapy", "treatment", y "management" y los términos booleanos AND y OR.

En PubMed se confeccionó la siguiente caja de búsqueda para encontrar la evidencia: ("dentin hypersensitivity" OR "dentin sensitivity" OR "tooth sensitivity") AND (therapy OR treatment) AND management. Luego se utilizó el concepto "Dentin Sensitivity" con terminología Mesh y se incluyó en la estrategia como se muestra a continuación: ("Dentin Sensitivity" [Mesh] OR "dentin hypersensitivity" OR "dentin sensitivity" OR "tooth sensitivity") AND (therapy OR treatment) AND management. Esta última caja de búsqueda se utilizó para buscar en PubMed Clinical Queries.

El resto de las bases de datos electrónicas no permiten una búsqueda extensa utilizando varias palabras, por lo que la búsqueda se llevó a cabo utilizando cada palabra clave por separado: "dentin hypersensitivity", "dentin sensitivity" y "tooth sensitivity".

Se realizó una crítica de la literatura científica donde se evaluó nivel de evidencia, calidad de reporte, riesgo de sesgo y ética. Para determinar el nivel de evidencia se utilizó el esquema propuesto por el Centre for Evidence-Based Medicine (CEBM) de Oxford(10). La calidad de reporte se evaluó por medio de cuestionarios y recomendaciones propuestos según el tipo de estudio, utilizando PRISMA 2009 para las revisiones sistemáticas y meta-análisis ${ }^{(11)}$ y CONSORT 2010 para los ensayos clínicos controlados aleatorizados ${ }^{(12)}$. Se estimó el riesgo de sesgo para cada ensayo clínico aleatorizado seleccionado en base a la pauta de The Cochrane Collaboration ${ }^{(13)}$. Por último, para la evaluación de la ética de los ensayos clínicos se consideraron los siguientes parámetros: existencia de consentimiento informado, aprobación por parte de un comité de ética, explicación al participante del objetivo del estudio y existencia o ausencia de conflictos de interés.

\section{RESULTADOS}

Una vez realizada la búsqueda en las cinco bases de datos mencionadas anteriormente con las estrategias descritas, se encontraron un total de 2.573 artículos. De este total, 756 artículos fueron seleccionados por título, 418 de ellos se encontraban duplicados, quedando 338 artículos potencialmente relevantes. Al aplicar los criterios de inclusión y exclusión 302 artículos fueron descartados, dejando un total de 36 artículos a ser incluidos; 29 de ellos corresponden a ensayos clínicos controlados aleatorizados y siete a revisiones sistemáticas.

Los ensayos clínicos encontrados se descomponen en; cinco $(17 \%)$ que probaron la eficacia de colutorios ${ }^{(14-18)}$, dos $(7 \%)$ que realizaron una comparación con dentífricos y colutorios ${ }^{(19,20)}$ y $22(76 \%)$ que evaluaron sólo dentífricos ${ }^{(21-42)}$

Todos los ensayos clínicos demostraron reducir la hipersensibilidad dentinaria con alguno de los agentes utilizados en comparación a la medición inicial. Al ser controlados aleatorizados todos tienen un nivel de evidencia $1 \mathrm{~b}$ según el centro de medicina basado en la evidencia de Oxford(10), cuyo grado de recomendación es A y su significado es extremadamente recomendable.

El resumen y resultado de cada ensayo clínico seleccionado se observa en la tabla 1, 2 y 3 ; donde se agruparon los estudios de colutorios, dentífricos y colutorios y sólo dentífricos respectivamente. Se observaron las variables: participantes por grupo, métodos diagnósticos, escala de evaluación subjetiva utilizada, agente intenvención y comparación, tiempo de seguimiento y resultados en cada artículo. Es posible notar la heterogeneidad en la metodolología al obsevar que ninguno de los parámetros evaluados fue igual para todos los estudios.

Con respecto a los agentes testeados encontramos (Gráfico 1): arginina, nitrato de potasio, fluoruro de potasio y de amina, citrato de potasio, oxalato de potasio, acetato de estroncio, nano hidroxiapatita, y fosfosilicato de calcio y sodio (CSPS) más conocido como Novamin ${ }^{\circledR}$ (GlaxoSmithKline Consumer Healthcare, Weybridge, Surrey, Reino Unido). Las sales de potasio fueron las más investigadas, siendo 19 ensayos los que evaluaron alguna de sus formas ${ }^{(14,16-20,25,26,28,29,32-38,40,41)}$. De ellas, el agente más testeado fue el nitrato de potasio, 15 estudios evaluaron su eficacia; dos de ellos en colutorios ${ }^{(14,18)}$, uno en una combinación de colutorio y dentífrico ${ }^{(19)}$, y 12 en dentífricos al $5 \%(25,28,29,33,35-41)$

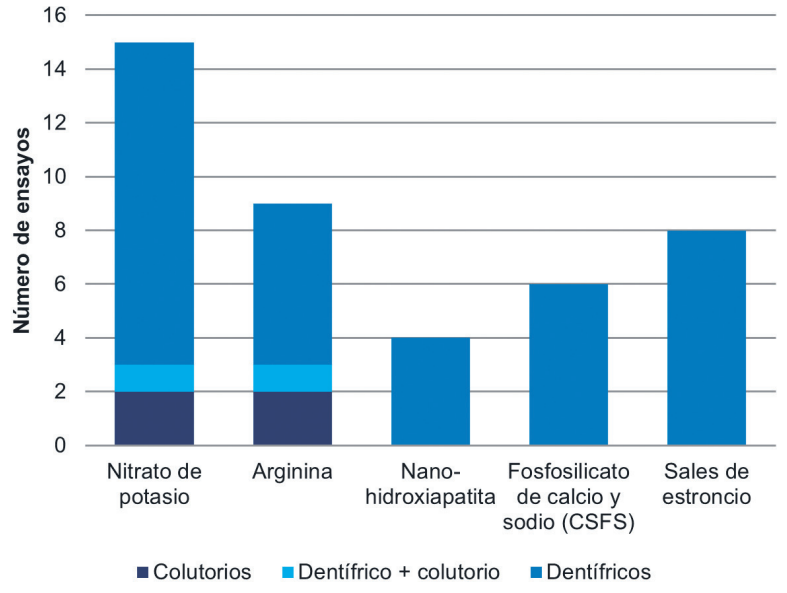

Gráfico 1. Principales agentes desensibilizantes testeados y forma de presentación.

Nueve estudios evaluaron la eficacia de la arginina; dos de ellos en colutorio con arginina al $0,8 \%$ y fluoruro de sodio al $0,05 \%{ }^{(14,15)}$; uno utilizando una combinación de colutorio - misma composición anterior - y un dentífrico con arginina al $8 \%{ }^{(19)}$; y seis sólo con dentífrico ${ }^{(21,23,26,31,36,37)}$

Cuatro estudios utilizaron la nano hidroxiapatita al $15 \%$ en dentífricos como intervención o comparación ${ }^{(21-24)}$, seis el fosfosilicato de calcio y sodio $^{(22,23,27,29,30,33)}$ y ocho los dentífricos con estroncio ${ }^{(21,26-28,35,39,40,42)}$.

En relación a la duración de los estudios, tres de ellos tuvieron una 
Tabla 1. Ensayos clínicos sobre colutorios.

\begin{tabular}{|c|c|c|c|c|c|c|}
\hline \multicolumn{7}{|c|}{ Ensayos clínicos sobre colutorios } \\
\hline Autor, año & $\begin{array}{l}\text { Participantes } \\
\text { por grupo }\end{array}$ & $\begin{array}{l}\text { Método } \\
\text { diagnóstico }\end{array}$ & $\begin{array}{l}\text { Evaluación } \\
\text { subjetiva }\end{array}$ & Intervención/ Comparación & $\begin{array}{l}\text { Medición } \\
\text { (semanas) }\end{array}$ & Resultados \\
\hline $\begin{array}{l}\text { Boneta, A. et } \\
\text { al. (2013) }\end{array}$ & 24 / 24 / 21 & $\begin{array}{c}\text { Estímulo táctil } \\
\text { (sonda Yeaple) } \\
\text { y aire }\end{array}$ & $\begin{array}{l}\text { Escala de } \\
\text { sensibilidad de } \\
\text { Schiff }\end{array}$ & $\begin{array}{c}\text { Arginina } 0,8 \% \text { y } 0,05 \% \text { NaF / KNO3 } \\
2,4 \% \text { y } 0,022 \% \mathrm{NaF} / \text { (Control -) } \\
0,05 \% \mathrm{NaF}\end{array}$ & Inicio, 2,4 y 6 & Arginina > KNO3 y control negativo \\
\hline $\begin{array}{l}\text { Hu, D. et al. } \\
\quad(2013)\end{array}$ & $45 / 45$ & $\begin{array}{c}\text { Estímulo táctil } \\
\text { (sonda Yeaple) } \\
\text { y aire }\end{array}$ & $\begin{array}{c}\text { Escala de } \\
\text { sensibilidad de } \\
\text { Schiff }\end{array}$ & $\begin{array}{c}\text { Arginina } 0,8 \% \text { y } 0,05 \% \mathrm{NaF} / \text { (control } \\
\text {-) } 0,05 \% \mathrm{NaF}\end{array}$ & Inicio, 2, 4 y 8 & Arginina > Control \\
\hline $\begin{array}{l}\text { Yates, RJ. et } \\
\text { al. (2004) }\end{array}$ & $46 / 45$ & Aire y agua fría. & EVA $(100 \mathrm{~mm})$ & $\begin{array}{l}\text { Fluoruro de potasio y de amina y } \\
\text { polímeros / Placebo }\end{array}$ & Inicio, 4 y 8 & Fluoruro de Potasio > Placebo \\
\hline $\begin{array}{l}\text { Yates, R. et al. } \\
\text { (1998) }\end{array}$ & $41 / 42$ & $\begin{array}{c}\text { Estímulo táctil } \\
\text { (sonda Yeaple) } \\
\text { y aire }\end{array}$ & $\begin{array}{l}\text { Escala de } \\
\text { evaluación } \\
\text { propia }\end{array}$ & $\begin{array}{l}\text { Citrato de K } 2 \% \text {, cloruro de } \\
\text { cetilpiridino 0,05\%, NaF 0,05\% y } \\
\text { fosfatos / Placebo }\end{array}$ & Inicio, 4 y 8 & Citrato de Potasio > Placebo \\
\hline $\begin{array}{l}\text { Gillam, DG. et } \\
\text { al. (1996) }\end{array}$ & $24 / 23$ & $\begin{array}{l}\text { Estímulo táctil } \\
\text { (sonda Yeaple) } \\
\text { y aire }\end{array}$ & EVA $(100 \mathrm{~mm})$ & KNO3 $3 \%$ y $0,05 \%$ NaF / 0,05 NaF & Inicio, 2 y 6 & Nitrato de Potasio > Fluoruro de Sodio \\
\hline
\end{tabular}

Tabla 2. Ensayos clínicos que evalúan eficacia de dentífricos y colutorios.

\begin{tabular}{|c|c|c|c|c|c|c|}
\hline \multicolumn{7}{|c|}{ Ensayos clínicos sobre dentífricos y colutorios } \\
\hline Autor, año & $\begin{array}{l}\text { Participantes } \\
\text { por grupo }\end{array}$ & $\begin{array}{l}\text { Método } \\
\text { diagnóstico }\end{array}$ & $\begin{array}{l}\text { Evaluación } \\
\text { subjetiva }\end{array}$ & Intervención/ Comparación & $\begin{array}{l}\text { Medición } \\
\text { (semanas) }\end{array}$ & Resultados \\
\hline $\begin{array}{l}\text { Boneta, A. } \\
(2013)\end{array}$ & $40 / 40 / 38$ & $\begin{array}{c}\text { Estímulo táctil } \\
\text { (sonda Yeaple) } \\
\text { y aire }\end{array}$ & $\begin{array}{l}\text { Escala de } \\
\text { sensibilidad de } \\
\text { Schiff }\end{array}$ & $\begin{array}{l}\text { Régimen de arginina: Dentífrico } \\
\text { arginina } 8 \% \text { y } 1450 \text { ppm } \mathrm{F}+\text { colutorio } \\
\text { arginina 0,8\% y 0,05\% F I } \\
\text { Régimen de K: Dentífrico KNO3 } 5 \% \text { y } \\
1450 \text { ppm de } \mathrm{F}+\text { colutorio de cloruro } \\
\text { de K 0,51\% y } 230 \mathrm{ppm} \text { de } \mathrm{F} \text { / (control } \\
\text {-) Dentífrico } 1450 \mathrm{ppm} \text { flúor + colutorio } \\
\text { sin flúor o arginina }\end{array}$ & Inicio, 2, 4 y 8 & $\begin{array}{c}\text { Régimen arginina }>\text { Potasio }>\text { Control } \\
\text { negativo }\end{array}$ \\
\hline $\begin{array}{l}\text { Sharma, D. et } \\
\text { al. (2013) }\end{array}$ & $74 / 76 / 73$ & $\begin{array}{c}\text { Estímulo táctil } \\
\text { (sonda Yeaple) } \\
\text { y aire }\end{array}$ & EVA $(100 \mathrm{~mm})$ & $\begin{array}{l}\text { Colutorio con KO 1,4\% + dentífrico } \\
\text { con NaF / (control +) Dentífrico con } \\
\text { KNO3 5\% / (control -): Dentífrico NaF }\end{array}$ & Inicio, 2 y 4 & $\begin{array}{c}\text { Colutorio } \mathrm{KO}=\text { Control positivo > } \\
\text { Control negativo }\end{array}$ \\
\hline
\end{tabular}

Tabla 3. Ensayos clínicos sobre dentífricos.

\begin{tabular}{|c|c|c|c|c|c|c|}
\hline \multicolumn{7}{|c|}{ Ensayos clínicos sobre colutorios } \\
\hline Autor, año & $\begin{array}{l}\text { Participantes } \\
\text { por grupo }\end{array}$ & $\begin{array}{c}\text { Método } \\
\text { diagnóstico }\end{array}$ & $\begin{array}{l}\text { Evaluación } \\
\text { subjetiva }\end{array}$ & Intervención/ Comparación & $\begin{array}{l}\text { Medición } \\
\text { (semanas) }\end{array}$ & Resultados \\
\hline $\begin{array}{l}\text { Douglas de } \\
\text { Oliviera, D. et al. } \\
(2016)\end{array}$ & $\begin{array}{l}8 \text { (División } \\
\text { boca en } \\
\text { cuadrantes) }\end{array}$ & Aire y agua fría. & EVA $(100 \mathrm{~mm})$ & $\begin{array}{l}\text { Acetato de estroncio y carbonato } \\
\text { de calcio / Carbonato de calcio y } \\
\text { arginina al } 8 \% \text { / n-HA / Placebo }\end{array}$ & $\begin{array}{c}\text { Inicio, } \\
\text { inmediato } \\
\text { aplicación, } 1 \text { y } \\
30 \text { días }\end{array}$ & $\begin{array}{c}\mathrm{n}-\mathrm{HA}>\text { Acetato de estroncio }>\text { Arginina } \\
>>\text { Placebo }\end{array}$ \\
\hline $\begin{array}{l}\text { Gopinath, N. et } \\
\text { al. (2015) }\end{array}$ & $18 / 18$ & $\begin{array}{l}\text { Estímulo táctil } \\
\text { (sonda de } \\
\text { examen), aire y } \\
\text { agua fría. }\end{array}$ & EVA $(100 \mathrm{~mm})$ & n-HA / CSPS $5 \%$ & Inicio y 4 & $\mathrm{n}-\mathrm{HA}=\mathrm{CSPS}$ \\
\hline $\begin{array}{l}\text { Jena, A. et al. } \\
\text { (2015) }\end{array}$ & - & $\begin{array}{l}\text { Estímulo táctil } \\
\text { (sonda de } \\
\text { examen) y aire }\end{array}$ & $\begin{array}{l}\text { Escala de } \\
\text { sensibilidad de } \\
\text { Schiff y EVA } \\
(100 \mathrm{~mm})\end{array}$ & CSPS 5\% / Arginina $8 \%$ / n-HA 15\% & $\begin{array}{l}\text { Inicio, } \\
\text { inmediato } \\
\text { aplicación, } \\
1 \text { y } 4\end{array}$ & $\mathrm{n}-\mathrm{HA}>$ Arginina $>\mathrm{CSPS}$ \\
\hline $\begin{array}{l}\text { Vano, M. et al. } \\
\text { (2014) }\end{array}$ & $35 / 35 / 35$ & $\begin{array}{l}\text { Estímulo táctil } \\
\text { (sonda de } \\
\text { examen) y aire. }\end{array}$ & $\begin{array}{c}\text { Escala de } \\
\text { sensibilidad de } \\
\text { Schiff y EVA } \\
(100 \mathrm{~mm})\end{array}$ & $\begin{array}{c}\text { n-HA 15\% sin Flúor / (Control +) NaF } \\
\text { / Placebo }\end{array}$ & Inicio, 2 y 4 & n-HA $>$ Control $+>$ Placebo \\
\hline $\begin{array}{l}\text { Kumari, M. et al. } \\
\qquad(2013)\end{array}$ & $30 / 30$ & Aire y agua fría. & EVA $(100 \mathrm{~mm})$ & $\begin{array}{c}\text { KNO3 y Spinacia oleracia (extracto } \\
\text { de espinaca) / Placebo }\end{array}$ & Inicio, 6 y 12 & KNO3 > Placebo \\
\hline
\end{tabular}


Tabla 3. Ensayos clínicos sobre dentífricos. (continuación)

\begin{tabular}{|c|c|c|c|c|c|c|}
\hline \multicolumn{7}{|c|}{ Ensayos clínicos sobre colutorios } \\
\hline Autor, año & $\begin{array}{l}\text { Participantes } \\
\text { por grupo }\end{array}$ & $\begin{array}{l}\text { Método } \\
\text { diagnóstico }\end{array}$ & $\begin{array}{l}\text { Evaluación } \\
\text { subjetiva }\end{array}$ & Intervención/ Comparación & $\begin{array}{l}\text { Medición } \\
\text { (semanas) }\end{array}$ & Resultados \\
\hline $\begin{array}{l}\text { West, N. et al. } \\
\qquad(2013)\end{array}$ & $42 / 38$ & $\begin{array}{c}\text { Estímulo táctil } \\
\text { (sonda Yeaple) } \\
\text { y aire }\end{array}$ & $\begin{array}{l}\text { Escala de } \\
\text { sensibilidad de } \\
\text { Schiff y EVA } \\
(100 \mathrm{~mm})\end{array}$ & $\begin{array}{l}\text { Acetato de estroncio } 8 \% \text { y } 1040 \\
\text { ppm de F / Arginina 8\% y } 1450 \text { ppm } \\
\text { de F. }\end{array}$ & Inicio y 3 días & Acetato de estroncio = Arginina \\
\hline $\begin{array}{l}\text { Ananthakrishna, } \\
\text { S. et al. (2012) }\end{array}$ & $20 / 20$ & Aire y agua fría. & EVA $(100 \mathrm{~mm})$ & $\begin{array}{c}\text { CSPS } 7,5 \% \text { / Cloruro de estroncio } \\
10 \%\end{array}$ & Inicio, 2, 4 y 6 & CSPS > Cloruro de estroncio \\
\hline $\begin{array}{l}\text { Liu, H. et al. } \\
\quad(2012)\end{array}$ & $38 / 41$ & $\begin{array}{c}\text { Estímulo táctil } \\
\text { (sonda Yeaple) } \\
\text { y aire. }\end{array}$ & $\begin{array}{l}\text { Escala de } \\
\text { sensibilidad de } \\
\text { Schiff }\end{array}$ & $\begin{array}{c}\text { Cloruro de estroncio } 2 \% \text { y KNO3 5\% } \\
\text { / Placebo }\end{array}$ & $\begin{array}{l}\text { Inicio, } 10 \\
\text { minutos y } 3 \\
\quad \text { días }\end{array}$ & Cloruro de estroncio > Placebo \\
\hline $\begin{array}{l}\text { Pradeep AR. et } \\
\text { al. (2012) }\end{array}$ & $\begin{array}{c}39 / 36 / 38 \\
\quad / 37\end{array}$ & Aire y agua fría. & EVA $(100 \mathrm{~mm})$ & $\begin{array}{c}\text { KNO3 5\% / CSPS 5\% / Fluoruro de } \\
\text { amina 3,85\% / Placebo }\end{array}$ & Inicio, 2 y 6 & $\begin{array}{c}\text { CSPS > Nitrato de Potasio }>\text { Fluoruro } \\
>\text { Placebo }\end{array}$ \\
\hline $\begin{array}{l}\text { Rajesh, KS. et } \\
\text { al. (2012) }\end{array}$ & $15 / 15$ & Aire y agua fría. & EVA $(100 \mathrm{~mm})$ & CSPS 5\% / Placebo & Inicio, 6 y 8 & CSPS > placebo \\
\hline $\begin{array}{l}\text { Kehua, Q. et al. } \\
\text { (2010) }\end{array}$ & $40 / 40 / 41$ & $\begin{array}{c}\text { Estímulo táctil } \\
\text { (sonda Yeaple) } \\
\text { y aire. }\end{array}$ & $\begin{array}{l}\text { Escala de } \\
\text { sensibilidad de } \\
\text { Schiff }\end{array}$ & $\begin{array}{l}\text { Arginina } 8 \%, 1450 \mathrm{ppm} \text { de } \\
\text { F y carbonato de Ca en alta } \\
\text { concentración (como agente } \\
\text { blanqueador) / Arginina } 8 \% \text { en base } \\
\text { de carbonato de } \mathrm{Ca} / 1450 \mathrm{ppm} \text { de } \\
\mathrm{F} \text { en base de carbonato de Ca. }\end{array}$ & Inicio, 2, 4 y 8 & $\begin{array}{l}\text { Arginina con alto contenido de } \\
\text { carbonato de calcio }=\text { arginina con } \\
\text { base }>\text { control positivo }\end{array}$ \\
\hline $\begin{array}{l}\text { Morales, O. et } \\
\text { al. (2010) }\end{array}$ & - & $\begin{array}{l}\text { Estímulo táctil } \\
\text { (Hand Held) } \\
\text { y aire }\end{array}$ & EVA $(100 \mathrm{~mm})$ & $\begin{array}{c}\text { Citrato de K al } 5,6 \% \text { y NaF 0,3\% / } \\
\text { Placebo }\end{array}$ & Inicio, 4 y 8 & Citrato de Potasio > Placebo \\
\hline $\begin{array}{l}\text { Pradeep, AR. et } \\
\text { al. (2010) }\end{array}$ & $36 / 37 / 37$ & $\begin{array}{l}\text { Chorro de aire } \\
\text { y agua fría. }\end{array}$ & EVA $(100 \mathrm{~mm})$ & $\begin{array}{c}\text { CSPS 5\% / (control + ) KNO3 5\% / } \\
\text { Placebo }\end{array}$ & Inicio, 2 y 6 & CSPS > KNO3 > Placebo \\
\hline $\begin{array}{l}\text { Prasad, KVV. Et } \\
\quad \text { al. (2010) }\end{array}$ & - & $\begin{array}{l}\text { Estímulo táctil } \\
\text { (sonda de } \\
\text { examen) y } \\
\text { térmico (sonda } \\
\text { termoeléc-trica) }\end{array}$ & $\begin{array}{l}\text { Escala de } \\
\text { evaluación } \\
\text { propia }\end{array}$ & $\begin{array}{l}\text { Citrato de potasio y de zinc, triclosan } \\
\text { y MFP / NaF, sílice, triclosan } 0,3 \% \text { y } \\
\text { copolimeros } 2 \%\end{array}$ & Inicio, 6 y 12 & Citrato de Potasio $>\mathrm{NaF}$ \\
\hline $\begin{array}{l}\text { Xing Ni, T. et al. } \\
\text { (2010) }\end{array}$ & $30 / 28$ & $\begin{array}{l}\text { Estímulo táctil } \\
\text { (sonda Yeaple) } \\
\text { y chorro de } \\
\text { aire. }\end{array}$ & $\begin{array}{l}\text { Escala de } \\
\text { sensibilidad de } \\
\text { Schiff }\end{array}$ & $\begin{array}{c}\text { Cloruro de estaño y } 1450 \text { ppm de F / } \\
\text { (control +) KNO3 5\%. }\end{array}$ & Inicio, 4 y 8 & Cloruro de estaño = KNO3 \\
\hline $\begin{array}{l}\text { Docimo, R. et al. } \\
\text { (2009) }\end{array}$ & $40 / 40$ & $\begin{array}{c}\text { Estímulo táctil } \\
\text { (sonda Yeaple) } \\
\text { y aire }\end{array}$ & $\begin{array}{l}\text { Escala de } \\
\text { sensibilidad de } \\
\text { Schiff }\end{array}$ & $\begin{array}{l}\text { Arginina al } 8 \% \text {, carbonato de calcio } \\
\text { y } 1450 \text { ppm de MFP / Dentífrico con } \\
\text { KNO3 5\% y } 1450 \text { ppm de NaF }\end{array}$ & $\begin{array}{l}\text { Inicio, } 1,2 \\
\quad 4, \text { y } 8\end{array}$ & Arginina $>$ KNO3 \\
\hline $\begin{array}{l}\text { Nathoo, S. et al. } \\
\qquad(2009)\end{array}$ & $42 / 41 / 42$ & $\begin{array}{c}\text { Estímulo táctil } \\
\text { (sonda Yeaple) } \\
\text { y aire }\end{array}$ & $\begin{array}{l}\text { Escala de } \\
\text { sensibilidad de } \\
\text { Schiff }\end{array}$ & $\begin{array}{c}\text { Arginina } 8 \% \text {, carbonato de calcio y } \\
1450 \text { ppm de F / KNO3 5\% y } 1450 \\
\text { ppm de NaF / } 1450 \text { ppm de F }\end{array}$ & $\begin{array}{l}\text { Inicio, } \\
\text { inmediato } \\
\text { aplicación y } 3 \\
\text { días. }\end{array}$ & Arginina $>$ KNO3 > Flúor \\
\hline \multirow{2}{*}{$\begin{array}{l}\text { Sowinsky, J. et } \\
\text { al. (2001) }\end{array}$} & $41 / 40$ & \multirow{2}{*}{$\begin{array}{c}\text { Estímulo táctil } \\
\text { (sonda Yeaple) } \\
\text { y aire. }\end{array}$} & \multirow{2}{*}{$\begin{array}{l}\text { Escala de } \\
\text { sensibilidad de } \\
\text { Schiff. }\end{array}$} & $\begin{array}{c}\text { KNO3 5\%, fluoruro de estaño y de } \\
\text { sodio (1450 ppm de F) / } 1450 \text { ppm } \\
\text { de F (NaF) }\end{array}$ & \multirow{2}{*}{ Inicio, 4 y 8} & \multirow{2}{*}{$\begin{array}{c}\mathrm{KNO} 3>\mathrm{NaF} ; \mathrm{KNO} 3>\text { citrato de } \\
\text { potasio }\end{array}$} \\
\hline & $54 / 51$ & & & $\begin{array}{l}\text { KNO3 } 5 \% \text {, fluoruro de estaño y de } \\
\text { sodio ( } 1450 \text { ppm de } \mathrm{F} \text { ) / cloruro de } \\
\text { K, triclosan y } 1450 \text { ppm de } \mathrm{F}(\mathrm{NaF})\end{array}$ & & \\
\hline $\begin{array}{l}\text { West, N. et al. } \\
\quad(1997)\end{array}$ & $38 / 37 / 37$ & $\begin{array}{l}\text { Estímulo táctil } \\
\text { (sonda de } \\
\text { examen) y aire. }\end{array}$ & EVA $(100 \mathrm{~mm})$ & $\begin{array}{c}\text { Acetato de estroncio y NaF / KNO3 } \\
\text { y NaF / NaF }\end{array}$ & Inicio, 2 y 6 & Acetato de estroncio $=\mathrm{KNO} 3=\mathrm{NaF}$ \\
\hline $\begin{array}{l}\text { Silverman G. et } \\
\quad \text { al. (1996) }\end{array}$ & $\begin{array}{c}52 / 53 / 63 \\
/ 62\end{array}$ & $\begin{array}{c}\text { Estímulo táctil } \\
\text { (sonda Yeaple) } \\
\text { y aire. }\end{array}$ & EVA $(100 \mathrm{~mm})$ & $\begin{array}{c}\mathrm{KNO} 35 \% \text { y NaF } 0,243 \% \text { / KNO3 } \\
5 \% \text { / Cloruro de estroncio } 10 \% / \\
\text { Placebo }\end{array}$ & Inicio, 2,4 y 8 & $\begin{array}{c}\mathrm{KNO} 3 \text { y NaF }=\mathrm{KNO} 3>\text { Cloruro de } \\
\text { estroncio }>\text { Placebo }\end{array}$ \\
\hline $\begin{array}{l}\text { Nagata, T. et al. } \\
\quad(1994)\end{array}$ & $18 / 18$ & $\begin{array}{c}\text { Estímulo táctil } \\
\text { (sonda Yeaple) } \\
\text { y aire. }\end{array}$ & $\begin{array}{l}\text { Escala de } \\
\text { evaluación } \\
\text { propia }\end{array}$ & KNO3 5\% / Placebo & $\begin{array}{l}\text { Inicio, } 2,4,8 \\
\quad \text { y } 12\end{array}$ & KNO3 > Placebo \\
\hline $\begin{array}{l}\text { Gillam, DG. et } \\
\text { al. (1992) }\end{array}$ & $20 / 20$ & $\begin{array}{c}\text { Estímulo táctil } \\
\text { (sonda Yeaple) } \\
\text { y aire. }\end{array}$ & EVA $(100 \mathrm{~mm})$ & $\begin{array}{l}\text { Cloruro de estroncio hexahidratado / } \\
\text { Cloruro de estroncio hexahidratado } \\
\qquad \sin \text { abrasivos }\end{array}$ & Inicio, 2, 4 y 8 & Ambos igualmente efectivos \\
\hline
\end{tabular}


duración de 12 semanas $^{(25,34,41)}, 12$ de ocho semanas ${ }^{(15-17,19,30-32,35,36,38,40,42)}$, seis de seis semanas ${ }^{(14,18,27,29,33,39)}$, cinco de cuatro semanas ${ }^{(20-24)}$ y tres de tres días ${ }^{(26,28,37)}$

Se utilizó la pauta de evaluación de riesgo de sesgo de The Cochrane Collaboration ${ }^{(13)}$ para medir la validez externa y confiabilidad de los ensayos clínicos aleatorizados. Del total de estudios, nueve (31\%) presentaron un bajo riesgo, $16(55 \%)$ moderado riesgo y $4(14 \%)$ un alto riesgo de sesgo.

Sólo cuatro (14\%) ensayos clínicos cumplieron con todos los aspectos éticos evaluados. Los parámetros que menos se cumplieron fueron (Gráfico 2): el reporte de la explicación verbal a los participantes y la inexistencia de conflictos de interés, sólo 11 artículos describieron cada punto.

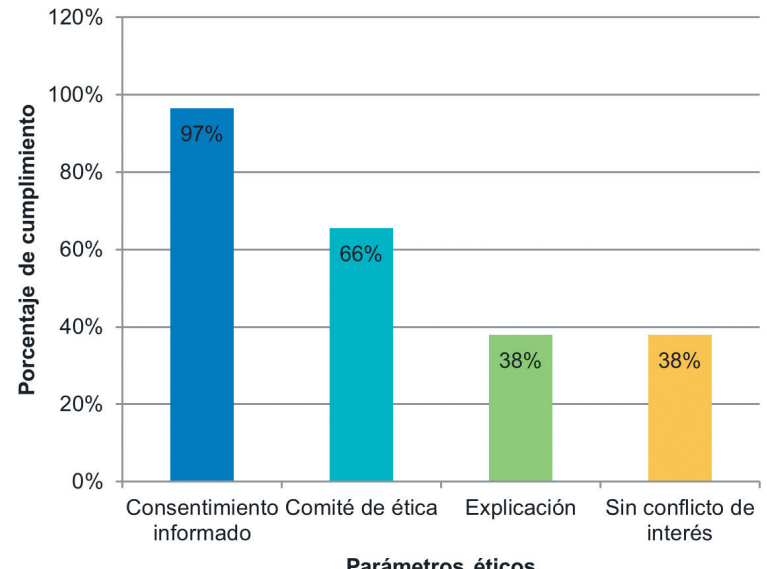

\section{Gráfico 2. Cumplimiento de parámetros éticos evaluados.}

Se seleccionaron tres artículos que corresponden a revisiones sistemáticas y meta-análisis ${ }^{(43-45)}$ y cuatro revisiones sistemáticas ${ }^{(46-49)}$; que fueron realizados entre los años 2012 y 2015; los resultados, así como la cantidad de artículos incluidos y los agentes a comparar se observan en la tabla 4 Los temas a tratar difieren en cada publicación, encontrando: dentífricos desensibilizantes en comparación a placebos u otros dentífricos ${ }^{(43,46)}$, pastas dentales que contienen sales de potasio ${ }^{(47,49)}$ y tres revisiones que evalúan la efectividad de los dentífricos con arginina ${ }^{(44,45,48)}$.

Se evaluó la calidad de reporte de cada artículo incluido en la presente revisión. De acuerdo con la pauta CONSORT del año 2010, 48\% de los ensayos clínicos controlados aleatorizados presentan una buena calidad de reporte, $48 \%$ una regular calidad y sólo un artículo, equivalente a un $3 \%$, presenta una mala calidad de reporte. En cuanto a las revisiones sistemáticas y meta-análisis evaluadas con el cuestionario PRISMA 2009, un artículo presentó una muy buena calidad de reporte $(14 \%)$, cinco una buena calidad ( $71 \%)$ y uno una regular calidad. No hubo artículos encontrados que tuvieran una mala calidad de reporte.

\section{DISCUSIÓN}

Uno de los principales problemas de los ensayos clínicos que evalúan la hipersensibilidad dentinaria corresponde a que se debe hacer una medición objetiva de un fenómeno subjetivo, el dolor. Para realizar estas mediciones se han usado métodos táctiles, evaporativos y térmicos con el propósito de estandarizar los estudios, pero no se sabe con certeza cuál de las mediciones es mejor ${ }^{(49)}$. La metodología de los ensayos clínicos seleccionados, a pesar de su alto nivel de evidencia, hace difícil la comparación, aunque se investigue acerca del mismo agente desensibilizante. Por ejemplo, en los ensayos incluidos se utilizaron distintos métodos diagnósticos y el intervalo de tiempo entre ambos estímulos variaba de no indicado a cinco, diez o 15 minutos.

Los agentes principalmente evaluados corresponden a: nitrato de potasio (KNO3), arginina, nano-hidroxiapatita (n-HA), acetato y cloruro de estroncio y fosfosilicato de calcio y sodio (CSPS). Todos, con la excepción del primero, son selladores de los túbulos dentinarios, siendo el nitrato de potasio un agente bloqueador de la conducción nerviosa ${ }^{(6-8)}$. En ninguna de las revisiones sistemáticas incluidas se menciona la intención de comparar entre los bloqueadores de la conducción con los selladores de los túbulos dentinarios.

En los 15 artículos incluidos que evalúan el nitrato de potasio, este

Tabla 4. Resultados de revisiones sistemáticas y meta-análisis.

\begin{tabular}{|c|c|c|c|}
\hline Autor, año & $\begin{array}{l}\text { Estudios } \\
\text { incluidos }\end{array}$ & Evaluación & Resultados \\
\hline \multicolumn{4}{|c|}{ Revisiones sistemáticas y meta-análisis } \\
\hline Bae, JK. et al. (2015) & 31 & Dentífricos desensibili-zantes vs. Placebo. & $\begin{array}{l}\text { Se recomienda el uso de pastas con potasio, fluoruro de estaño, } \\
\text { potasio y fluoruro de estaño, fosfosilicato de calcio y sodio y arginina en } \\
\text { comparación al placebo, no así el uso de pastas con estroncio. }\end{array}$ \\
\hline Poulsen, S. et al. (2012) & 6 & $\begin{array}{l}\text { Dentífricos con sales de potasio vs. } \\
\text { Placebo. }\end{array}$ & $\begin{array}{c}\text { Existen diferencias significativas con el uso de sales de potasio al } \\
\text { estímulo táctil, evaporativo y térmico, pero no para la evaluación } \\
\text { subjetiva. }\end{array}$ \\
\hline Talioti, E. et al. (2014) & 5 & $\begin{array}{l}\text { Dentífricos con fosfosilicato de calcio y } \\
\text { sodio, fosfato de calcio, nano hidroxiapatita } \\
\text { y fosfopéptido de caseína. }\end{array}$ & $\begin{array}{c}\text { El CSFS y el fosfato de calcio pudiesen ser efectivos en aliviar la HSD, } \\
\text { pero se requieren mayor cantidad de estudios. No se puede concluir } \\
\text { respecto del fosfopéptido de caseína y la nano-hidroxiapatita porque no } \\
\text { se incluyeron estudios en la revisión. }\end{array}$ \\
\hline Karim, BFA. et al. (2013) & 7 & $\begin{array}{l}\text { Dentífricos con estroncio y sales de } \\
\text { potasio. }\end{array}$ & $\begin{array}{l}\text { Ambos agentes han demostrado reducir la HSD frente a placebos, sin } \\
\text { embargo, la evidencia es insuficiente para determinar su efectividad. }\end{array}$ \\
\hline Sharif, M. et al. (2013) & 2 & Dentífricos con arginina vs. Placebo. & $\begin{array}{l}\text { Existe un potencial rol de la arginina para el control de la HSD. Se } \\
\text { recomienda realizar más y mejores ensayos clínicos. }\end{array}$ \\
\hline
\end{tabular}


demostró ser un agente efectivo para la sensibilidad. En dentífricos siempre se usó en concentración al $5 \%$, mientras que en colutorios variaba de 2,4 a $5 \%$. Al compararlo con otros agentes, demostró ser más afectivo que el cloruro de estroncio y el placebo según Silverman et al. ${ }^{(40)}$; e igualmente efectivo que el acetato de estroncio y cloruro de estaño de acuerdo con West et al. ${ }^{(39)}$ y Xing Ni et al. ${ }^{(35)}$ respectivamente. Según Docimo et al. y Nathoo et al. demostró ser menos efectivo que la arginina al $8 \%$ en dentífricos ${ }^{(36,37)}$ y en colutorios al $0,8 \%$ de acuerdo a Boneta et al. ${ }^{(14,19)}$. Por último, en comparación al fosfosilicato de calcio y sodio al $5 \%$ fue menos efectivo según el estudio de Pradeep et al. en el año 2010 y $2012^{(29,33)}$. A diferencia de la primera revisión sistemática sobre las pastas dentales con potasio realizada por Poulsen et al. ${ }^{(49)}$; las revisiones de Bae et al. y Karim et al. describen positivamente su eficacia como agente desensibilizante ${ }^{(43,47)}$; sin embargo, todas concuerdan que la calidad de la evidencia es insuficiente para recomendar su uso.

Nueve ensayos clínicos evaluaron la arginina. Se usó la concentración al $8 \%$ en dentífricos y al $0,8 \%$ en colutorios. Cuatro revisiones sistemáticas elaboradas por Bae et al., Yan et al., Magno et al. y Sharif et al. ${ }^{(43-45,48)}$; concuerdan que la arginina es efectiva como agente desensibilizante. Es más, los resultados indican que es significativamente superior al placebo luego de la medición inmediata a la aplicación ${ }^{(44,48)}$; que las pastas con potasio en las mediciones a los tres días y ocho semanas ${ }^{(44)} ; \mathrm{y}$ que los dentífricos con estroncio en las mediciones a las ocho semanas ${ }^{(44,45)}$.

Tres estudios, dos de Boneta et al. y uno de Hu et al., avalan su uso en colutorios e indican que es más efectivo que el nitrato de potasio y el control negativo ${ }^{(14,15,19)}$. Douglas de Oliviera et al. y Jena et al. ${ }^{(21,23)}$ indican que es menos efectiva que la nano-hidroxiapatita a las cuatro semanas de uso.

Los ensayos sobre la nano-hidroxiapatita tuvieron siempre una duración de cuatro semanas, utilizan una concentración al $15 \%$ y su forma de presentación es exclusivamente en dentífricos. Los cuatro concuerdan que la n-HA es un agente efectivo en la reducción de la HSD ${ }^{(21-24)}$. Cabe destacar que este agente no fue evaluado en ninguna de las revisiones sistemáticas seleccionadas.

Los seis estudios que evalúan el fosfosilicato de calcio y sodio usan concentraciones de dentífricos al $5 \%$. Dos revisiones sistemáticas, una de ellas meta-análisis desarrollado por Bae et al. ${ }^{(43)}$, indican que es un efectivo agente desensibilizante, sin embargo señalan que se requieren más estudios y de mejor calidad para dar conclusiones más certeras ${ }^{(43,46)}$. Cuatro ensayos clínicos sostienen que su uso es más efectivo que el cloruro de estroncio ${ }^{(27)}$, nitrato de potasio y placebo ${ }^{(29,30,33)}$. El estudio de Gopinath et al. ${ }^{(22)}$ indica que la nano-hidroxiapatita tiene igual efectividad a las cuatro semanas de uso, mientras que el de Jena et al. ${ }^{(23)}$ indica que la n-HA y arginina producen un alivio sintomático mayor a la medición inmediata y a las cuatro semanas de uso.

El completo alivio de la sintomatología no se observó al utilizar ninguno de los agentes, pero si es evidente la disminución del dolor luego de la aplicación del tratamiento e incluso frente a los placebos o controles negativos.

Es habitual encontrar en la literatura cuando se refiere a la hipersensibilidad dentinaria, el efecto placebo, como lo menciona West et al. en $1997^{(39)}$. Este consiste en la compleja interacción de factores psicológicos y fisiológicos que dependen de la relación odontólogo-paciente, donde ambas partes desean el alivio de la sintomatología o el éxito de un tratamiento ${ }^{(39,50)}$.

A pesar del uso de un componente aleatorio y de la estratificación de los grupos para homogeneizar las muestras, los voluntarios a menudo intentan complacer a los clínicos, pudiendo alcanzar el efecto deseado en las pastas con el componente activo ${ }^{(39)}$. Este fenómeno se ha evidenciado y evaluado en 11 de los ensayos clínicos revisados ${ }^{(16,17,21,24,25,28-30,33,40,41)}$.

\section{CONCLUSIÓN}

De acuerdo con la evidencia se podría recomendar el uso de nitrato y citrato de potasio, arginina, fosfosilicato de calcio y sodio y nano-hidroxiapatita en dentífricos para el tratamiento de la hipersensibilidad, aunque sin duda se requieren más ensayos clínicos controlados aleatorizados homogéneos entre sí y con menor sesgo, que permitan realizar recomendaciones con evidencia fundamentada para su uso a largo y corto plazo. En cuanto a los colutorios, aunque los estudios incluidos demuestran que disminuyen la sensibilidad, sólo se encontraron ensayos que evaluaran la efectividad con nitrato de potasio y arginina. Es necesaria la formulación de nuevos colutorios para probar la eficacia de otros agentes desensibilizantes.

Ninguno de los agentes evaluados logró el completo alivio de la sintomatología sólo se observó una disminución del dolor y aunque sea posible asumir el valor terapéutico de algunos agentes, el efecto placebo podría enmascarar los resultados.

Para futuras revisiones se recomienda incluir artículos que utilicen los mismos métodos diagnósticos y evalúen un menor número de agentes desensibilizantes para que a pesar de encontrar un menor número de resultados, los ensayos clínicos sean homogéneos y sea posible obtener conclusiones con mayor relevancia clínica.

\section{CONFLICTO DE INTERESES Y FUENTE DE FINANCIAMIENTO}

Los autores declaran no tener ningún conflicto de intereses.

\section{Bibliografía}

1. Canadian Advisory Board on Dentin Hypersensitivity. Consensus-based recommendations for the diagnosis and management of dentin hypersensitivity. $J$ Can Dent Assoc. 2003;69(4):221-6.

2. Lin $\mathrm{YH}$, Gillam DG. The prevalence of root sensitivity following periodontal therapy: a systematic review. Int J Dent. 2012;2012:e407023.

3. Orchardson R, Collins WJ. Thresholds of hypersensitive teeth to 2 forms of controlled stimulation. J Clin Periodontol. 1987;14(2):68-73.

4. Gillam DG. Current diagnosis of dentin hypersensitivity in the dental office: an overview. Clin Oral Investig. 2013;17 Suppl 1:S21-9.

5. Holland GR, Narhi MN, Addy M, Gangarosa L, Orchardson R. Guidelines for the design and conduct of clinical trials on dentine hypersensitivity. J Clin Periodontol. 1997;24(11):808-13.

6. Orchardson R, Gillam DG. Managing dentin hypersensitivity. J Am Dent Assoc. 2006;137(7):990-998; quiz 1028-1029.

7. Bartold PM. Dentinal hypersensitivity: a review. Aust Dent J. 2006;51(3):212-218; quiz 276.

8. Taha S, Clarkson BH. Clinician's guide to the diagnosis and management of tooth sensitivity. Springer Science \& Business Media; 2014. $112 p$

9. West NX, Seong J, Davies M. Management of dentine hypersensitivity: efficacy of professionally and self-administered agents. J Clin Periodontol. 2015;42 Suppl 16:S256-302.

10. Primo J. Niveles de evidencia y grados de recomendación (I/II). Enferm Inflamatoria Intest AI Día. 2003;2(2):39-42.

11. Moher D, Liberati A, Tetzlaff J, Altman DG, Group TP. Preferred reporting items for systematic reviews and meta-analyses: the PRISMA statement. PLOS Med. 2009;6(7):e1000097.

12. Cobos-Carbo A, Augustovski F. Declaración CONSORT 2010: actualización de la lista de comprobación para informar ensayos clínicos aleatorizados de grupos paralelos. Med Clínica. 2011;137(5):213-5

13. Higgins J, Green, S. Cochrane handbook for systematic reviews of interventions. 2011 [citado 16 de agosto de 2016]; Version 5.1.0 [updated March 2011]. Disponible en: www.handbook.cochrane.org

14. Elías Boneta AR, Galán Salás RM, Mateo LR, Stewart B, Mello S, Arvanitidou $\mathrm{LS}$, et al. Efficacy of a mouthwash containing $0.8 \%$ arginine, PVM/MA copolymer, pyrophosphates, and $0.05 \%$ sodium fluoride compared to a commercial mouthwash containing $2.4 \%$ potassium nitrate and $0.022 \%$ sodium fluoride and a control mouthwash containing $0.05 \%$ sodium fluoride on dentine hypersensitivity: a sixweek randomized clinical study. J Dent. 2013;41 Suppl 1:S34-41.
15. Hu D, Stewart B, Mello S, Arvanitidou L, Panagakos F, De Vizio W, et al. Efficacy of a mouthwash containing $0.8 \%$ arginine, PVM/MA copolymer, pyrophosphates, and $0.05 \%$ sodium fluoride compared to a negative control mouthwash on dentin hypersensitivity reduction. A randomized clinical trial. J Dent. 2013;41 Suppl 1:S2633.

16. Yates RJ, Newcombe RG, Addy M. Dentine hypersensitivity: a randomised double-blind placebo-controlled study of the efficacy of a fluoride-sensitive teeth mouthrinse. J Clin Periodontol. 2004;31(10):885-9.

17. Yates R, West N, Addy M, Marlow I. The effects of a potassium citrate, cetylpyridinium chloride, sodium fluoride mouthrinse on dentine hypersensitivity, plaque and gingivitis. A placebo-controlled study. J Clin Periodontol. 1998;25(10):813-20.

18. Gillam DG, Bulman JS, Jackson RJ, Newman HN. Efficacy of a potassium nitrate mouthwash in alleviating cervical dentine sensitivity (CDS). J Clin Periodontol. 1996;23(11):993-7.

19. Elías Boneta AR, Ramirez K, Naboa J, Mateo LR, Stewart B, Panagokos F, et al. Efficacy in reducing dentine hypersensitivity of a regimen using a toothpaste containing $8 \%$ arginine and calcium carbonate, a mouthwash containing $0.8 \%$ arginine, pyrophosphate and PVM/MA copolymer and a toothbrush compared to potassium and negative control regimens: an eight-week randomized clinical trial. J Dent. 2013;41 Suppl 1:S42-9.

20. Sharma D, McGuire JA, Gallob JT, Amini P. Randomised clinical efficacy trial of potassium oxalate mouthrinse in relieving dentinal sensitivity. J Dent. 2013;41 Suppl 4:S40-8.

21. Douglas de Oliveira DW, Oliveira ES de, Mota AFM, Pereira VHU, Bastos VO, Glória JCR, et al. Effectiveness of three desensitizing dentifrices on cervical dentin hypersensitivity: a pilot clinical trial. J Int Acad Periodontol. 2016;18(2):57-65.

22. Gopinath NM, John J, Nagappan N, Prabhu S, Kumar ES. Evaluation of dentifrice containing nano-hydroxyapatite for dentinal hypersensitivity: a randomized controlled trial. J Int Oral Health. 2015;7(8):118-22.

23. Jena A, Shashirekha G. Comparison of efficacy of three different desensitizing agents for in-office relief of dentin hypersensitivity: a 4 weeks clinical study. J Conserv Dent. 2015;18(5):389-93.

24. Vano M, Derchi G, Barone A, Covani U. Effectiveness of nano-hydroxyapatite toothpaste in reducing dentin hypersensitivity: a double-blind randomized controlled trial. Quintessence Int. 2014;45(8):703-11.

25. Kumari M, Naik SB, Rao NS, Martande SS, Pradeep AR. Clinical efficacy of a herbal dentifrice on dentinal hypersensitivity: a randomized controlled clinical trial. Aust Dent J. 2013;58(4):483-90. 
26. West N, Newcombe RG, Hughes N, Mason S, Maggio B, Sufi F, et al. A 3-day randomised clinical study investigating the efficacy of two toothpastes, designed to occlude dentine tubules, for the treatment of dentine hypersensitivity. J Dent. 2013;41(2):187-94.

27. Ananthakrishna S, Raghu TN, Koshy S, Kumar N. Clinical evaluation of the efficacy of bioactive glass and strontium chloride for treatment of dentinal hypersensitivity. J Interdiscip Dent. 2012;2(2):92-7.

28. Liu H, Hu D. Efficacy of a commercial dentifrice containing $2 \%$ strontium chloride and $5 \%$ potassium nitrate for dentin hypersensitivity: a 3-day clinical study in adults in China. Clin Ther. 2012;34(3):614-22.

29. Pradeep AR, Agarwal E, Naik SB, Bajaj P, Kalra N. Comparison of efficacy of three commercially available dentifrices on dentinal hypersensitivity: a randomized clinical trial. Aust Dent J. 2012;57(4):429-34

30. Rajesh KS, Hedge S, Arun Kumar MS, Shetty DG. Evaluation of the efficacy of a $5 \%$ calcium sodium phosphosilicate (Novamin) containing dentifrice for the relie of dentinal hypersensitivity: a clinical study. Indian J Dent Res. 2012;23(3):363-7.

31. Que K, Fu Y, Lin L, Hu D, Zhang YP, Panagakos FS, et al. Dentin hypersensitivity reduction of a new toothpaste containing $8.0 \%$ arginine and $1450 \mathrm{ppm}$ fluoride: an 8-week clinical study on Chinese adults. Am J Dent. 2010;23 Spec No A:28A-35.

32. Morales OMR, Londoño W, Ortiz LGG, Patiño EG, Blandón N, García N. Eficacia de una crema dental para dientes sensibles con citrato de potasio al $5.6 \%$ y fluoruro de sodio al $0.3 \%$ : ensayo clínico aleatorizado controlado. Rev Fac Odontol Univ Antioquia. 2010;21(2):186-97.

33. Pradeep AR, Sharma A. Comparison of clinical efficacy of a dentifrice containing calcium sodium phosphosilicate to a dentifrice containing potassium nitrate and to a placebo on dentinal hypersensitivity: a randomized clinical trial. J Periodontol. 2010;81(8):1167-73

34. Prasad KVV, Sohoni R, Tikare S, Yalamalli M, Rajesh G, Javali SB. Efficacy of two commercially available dentifrices in reducing dentinal hypersensitivity. Indian J Dent Res. 2010;21(2):224-30.

35. Ni LX, He T, Chang A, Sun L. The desensitizing efficacy of a novel stannouscontaining sodium fluoride dentifrice: an 8-week randomized and controlled clinical trial. Am J Dent. 2010;23 Spec No B:17B-21.

36. Docimo R, Montesani L, Maturo P, Costacurta M, Bartolino M, Zhang YP, et al. Comparing the efficacy in reducing dentin hypersensitivity of a new toothpaste containing $8.0 \%$ arginine, calcium carbonate, and $1450 \mathrm{ppm}$ fluoride to a benchmark commercial desensitizing toothpaste containing $2 \%$ potassium ion: an eight-week clinical study in Rome, Italy. J Clin Dent. 2009;20(4):137-43.

37. Nathoo S, Delgado E, Zhang YP, DeVizio W, Cummins D, Mateo LR. Comparing the efficacy in providing instant relief of dentin hypersensitivity of a new toothpaste containing $8.0 \%$ arginine, calcium carbonate, and $1450 \mathrm{ppm}$ fluoride relative to a benchmark desensitizing toothpaste containing $2 \%$ potassium ion and $1450 \mathrm{ppm}$ fluoride, and to a control toothpaste with $1450 \mathrm{ppm}$ fluoride: a three-day clinical study in New Jersey, USA. J Clin Dent. 2009;20(4):123-30.
38. Sowinski J, Ayad F, Petrone M, DeVizio W, Volpe A, Ellwood R, et al. Comparative investigations of the desensitising efficacy of a new dentifrice. J Clin Periodontol. 2001;28(11):1032-6.

39. West NX, Addy M, Jackson RJ, Ridge DB. Dentine hypersensitivity and the placebo response. A comparison of the effect of strontium acetate, potassium nitrate and fluoride toothpastes. J Clin Periodontol. 1997;24(4):209-15.

40. Silverman G, Berman E, Hanna CB, Salvato A, Fratarcangelo P, Bartizek $\mathrm{RD}$, et al. Assessing the efficacy of three dentifrices in the treatment of dentinal hypersensitivity. J Am Dent Assoc. 1996;127(2):191-201.

41. Nagata T, Ishida H, Shinohara H, Nishikawa S, Kasahara S, Wakano $Y$, et al. Clinical evaluation of a potassium nitrate dentifrice for the treatment of dentinal hypersensitivity. J Clin Periodontol. 1994;21(3):217-21.

42. Gillam DG, Newman HN, Davies EH, Bulman JS. Clinical efficacy of a low abrasive dentifrice for the relief of cervical dentinal hypersensitivity. J Clin Periodontol. 1992;19(3):197-201.

43. Bae J-H, Kim Y-K, Myung S-K. Desensitizing toothpaste versus placebo for dentin hypersensitivity: a systematic review and meta-analysis. J Clin Periodontol. 2015;42(2):131-41

44. Yan B, Yi J, Li Y, Chen Y, Shi Z. Arginine-containing toothpastes for dentin hypersensitivity: systematic review and meta-analysis. Quintessence Int 2013;44(9):709-23

45. Magno MB, Nascimento GCR, Da Penha NKS, Pessoa OF, Loretto SC, Maia LC. Difference in effectiveness between strontium acetate and arginine-based toothpastes to relieve dentin hypersensitivity. A systematic review. Am J Dent 2015;28(1):40-4

46. Talioti E, Hill R, Gillam DG. The efficacy of selected desensitizing OTC products: a systematic review. ISRN Dent. 2014;27:865761

47. Karim BFA, Gillam DG. The efficacy of strontium and potassium toothpastes in treating dentine hypersensitivity: a systematic review. Int J Dent. 2013;2013:573258 48. Sharif MO, Iram S, Brunton PA. Effectiveness of arginine-containing toothpastes in treating dentine hypersensitivity: a systematic review. J Dent. 2013;41(6):483-92. 49. Poulsen S, Errboe M, Lescay Mevil Y, Glenny A-M. Potassium containing toothpastes for dentine hypersensitivity. Cochrane Database Syst Rev. 2006;(3):CD001476.

50. Trowbridge HO, Silver DR. A review of current approaches to in-office management of tooth hypersensitivity. Dent Clin North Am. 1990;34(3):561-81. 